\title{
Absolutely Maximally Entangled States of Seven Qubits Do Not Exist
}

\author{
Felix Huber, ${ }^{1}$ Otfried Gühne, ${ }^{1}$ and Jens Siewert ${ }^{2,3}$ \\ ${ }^{1}$ Naturwissenschaftlich-Technische Fakultät, Universität Siegen, 57068 Siegen, Germany \\ ${ }^{2}$ Departamento de Química Física, Universidad del País Vasco UPV/EHU, E-48080 Bilbao, Spain \\ ${ }^{3}$ IKERBASQUE Basque Foundation for Science, E-48013 Bilbao, Spain
}

(Received 6 November 2016; published 17 May 2017)

\begin{abstract}
Pure multiparticle quantum states are called absolutely maximally entangled if all reduced states obtained by tracing out at least half of the particles are maximally mixed. We provide a method to characterize these states for a general multiparticle system. With that, we prove that a seven-qubit state whose three-body marginals are all maximally mixed, or equivalently, a pure $((7,1,4))_{2}$ quantum error correcting code, does not exist. Furthermore, we obtain an upper limit on the possible number of maximally mixed three-body marginals and identify the state saturating the bound. This solves the seven-particle problem as the last open case concerning maximally entangled states of qubits.
\end{abstract}

DOI: 10.1103/PhysRevLett.118.200502

Introduction.-Multiparticle entanglement is central for the understanding of the possible quantum advantages in metrology or information processing. When investigating multiparticle entanglement as a resource, the question arises regarding which quantum states are most entangled. For a pure multiparticle quantum state maximal entanglement is present across a bipartition if the smaller of the two corresponding reduced systems is maximally mixed. It is, then, a natural question to ask whether or not there exist quantum states for any number of parties $n$, such that all of their reductions to $\lfloor n / 2\rfloor$ parties are maximally mixed $[1,2]$. If this is the case, maximal entanglement is present across all bipartitions and, accordingly, these states are also known as absolutely maximally entangled (AME) states [3-18]. These states have been shown to be a resource for open-destination and parallel teleportation [10], for threshold quantum secret sharing schemes [12], and are a type of quantum error correcting codes [3].

If the local dimension is chosen large enough, AME states always exist [12]. For qubits, however, the situation is only partially resolved. The three-qubit Greenberger-HorneZeilinger state is an AME state since all the single-qubit reduced states are maximally mixed. For four qubits, it was shown that AME states do not exist [2] and best approximations of AME states (where not all reduced states are maximally mixed) have been presented [8]. Five- and sixqubit AME states are known [3,5,7]. These can be represented as graph states and correspond to additive or stabilizer codes used in quantum error correction $[3,19]$. For more than eight qubits, AME states do not exist [3,20-22].

Despite many attempts, the case of seven qubits remained unresolved. Numerical results give some evidence for the absence of an AME state [5-7]. By exhaustive search, it was shown that such a state could not have the form of a stabilizer state [19]. Nevertheless, some approximation has been presented by making many, but not all, three-body marginals maximally mixed $[9,23]$.
As we have mentioned, AME states are a type of pure quantum error correcting codes (QECCs), having the maximal distance allowed by the Singleton bound [24]. In particular, AME states of $n$ parties having local dimension $D$ each correspond to a pure QECC in $\left(\mathbb{C}^{D}\right)^{\otimes n}$ of distance $\lfloor n / 2\rfloor+1$, denoted by $((n, 1,\lfloor n / 2\rfloor+1))_{D}$. Often, but not always, bounds on so-called nonadditive (i.e., nonstabilizer) codes coincide with those for additive (stabilizer) codes. The seven qubit AME state would-if it existed - be one of the few examples where a nonadditive code outperformed an additive one. This possibility was noted already in a seminal article by Calderbank et al. [25]. Up to $n=30$, there are only three other instances known where this could be the case for one-dimensional codes on qubits [26].

In this Letter, we provide a method for characterizing AME states and their approximations, making use of the Bloch representation [27]. The usefulness of this tool may be surprising at first sight, as the Bloch representation is designed to be a tool for mixed states. We were motivated to choose this approach by the fact that monogamy equalities $[8,28]$ directly signal the nonexistence of a four-qubit AME state, and the natural framework for deriving the monogamy equalities appears to be the Bloch representation [28].

The Bloch representation.-Any $n$-qubit state can be written as

$$
\varrho=\frac{1}{2^{n}} \sum_{\alpha_{1}, \ldots, \alpha_{n}} r_{\alpha_{1}, \ldots, \alpha_{n}} \sigma_{\alpha_{1}} \otimes \cdots \otimes \sigma_{\alpha_{n}},
$$

where the $\left\{\alpha_{1}, \ldots, \alpha_{n}\right\} \in\{0, x, y, z\}$ label combinations of the four Pauli matrices. For simplicity, we group the terms according to their weight, that is, their number of nontrivial (Pauli) operators. Let $P_{j}$ be the sum over terms of weight $j$, then, the state can be written as

$$
\varrho=\frac{1}{2^{n}}\left(\mathbb{1}^{\otimes n}+\sum_{j=1}^{n} P_{j}\right) .
$$


We denote by $P_{j}^{(V)}$ a subset of $P_{j}$, where $V$ further specifies its support; i.e., its nontrivial terms are located on the subsystems in $V$. To give an example, a state of three qubits reads

$$
\varrho=\frac{1}{2^{3}}\left(\mathbb{1}^{\otimes 3}+\sum_{j=1}^{3} P_{1}^{(j)}+\sum_{1 \leq k<l \leq 3} P_{2}^{(k l)}+P_{3}\right),
$$

where, e.g., $\quad P_{2}^{(12)}=\sum r_{\alpha_{1}, \alpha_{2}, 0} \sigma_{\alpha_{1}} \otimes \sigma_{\alpha_{2}} \otimes \mathbb{1} \quad$ and $\quad \alpha_{1}$, $\alpha_{2} \neq 0$. When tracing out the third qubit, one drops the terms $P_{3}, P_{2}^{(13)}, P_{2}^{(23)}$, and $P_{1}^{(3)}$, as they do not contain an identity in the third subsystem. Also, the normalization prefactor is multiplied by the dimension of the parties over which the partial trace was performed, resulting in

$$
\operatorname{tr}_{\{3\}}[\varrho] \otimes \mathbb{1}=\frac{1}{2^{2}}\left(\mathbb{1}^{\otimes 3}+P_{1}^{(1)}+P_{1}^{(2)}+P_{2}^{(12)}\right) .
$$

Accordingly, a three-qubit state having maximally mixed one-body reduced density matrices does not have terms of weight one, the terms $P_{1}^{(j)}$ are absent. Similarly, in $n$-qubit AME states, all operators $P_{j}$ with $1 \leq j \leq\lfloor n / 2\rfloor$ vanish.

Our discussion further rests on recognizing what terms may appear in the squared state $Q^{2}$. For this, consider two terms $M$ and $N$, both appearing in the Bloch expansion of the state. For computing $Q^{2}$, the anticommutator $\{M, N\}$ is required, and we state the following observation regarding its weight.

Lemma 1 (parity rule).-Let $M, N$ be Hermitian operators proportional to $n$-fold tensor products of single-qubit Pauli operators, $\quad M=c_{M} \sigma_{\mu_{1}} \otimes \cdots \otimes \sigma_{\mu_{n}}, \quad N=c_{N} \sigma_{\nu_{1}} \otimes \cdots \otimes \sigma_{\nu_{n}}$, where $c_{M}, c_{N} \in \mathbb{R}$. Let us denote their weights, that is, their number of nontrivial Pauli operators in their tensor expansion, by $|M|$ and $|N|$. Then, if the anticommutator $\{M, N\}$ does not vanish, its weight $|\{M, N\}|$ fulfills

$$
|\{M, N\}|=|M|+|N| \bmod 2 .
$$

Proof.-The product $M N$ and thus $\{M, N\}$ has, at most, a weight of $|M|+|N|$. This is attained, if the supports of $M$ and $N$ are disjoint. Each pair of equal, but nonzero indices $\mu_{j}=\nu_{j}$ corresponds to some overlap of the supports and reduces the maximal weight $|M|+|N|$ by two. In contrast, if a pair of nonzero indices are not equal (e.g., $\mu_{j} \neq \nu_{j}$ ), the product $M N$ contains the term $\sigma_{\mu_{j}} \sigma_{\nu_{j}}=$ $i \epsilon_{\mu_{j} \nu_{j} \chi} \sigma_{\chi}$. Consequently, for each such pair $|M|+|N|$ is reduced by only one. If an odd number of such pairs exists, the anticommutator has to vanish, as it is Hermitian. So, such pairs have to occur an even number of times, which proves the claim.

We can summarize the behavior of the weights of $M$ and $N$ and their anticommutator as follows:

$$
\begin{aligned}
\{\text { even, even }\} & \rightarrow \text { even }, \\
\{\text { odd, odd }\} & \rightarrow \text { even }, \\
\{\text { even, odd }\} & \rightarrow \text { odd } .
\end{aligned}
$$

It follows that an analogous behavior holds for the $P_{j}$. If $j$ and $k$ are either both even or both odd, the anticommutator $\left\{P_{j}, P_{k}\right\}$ can only contribute to the $P_{l}$ where $l$ is even. Similarly, if $j$ is even and $k$ is odd, it only contributes to the $P_{l}$ having odd $l$.

Properties of AME state reductions.-Recall that, for a pure $n$-party state $|\psi\rangle_{A B}$ consisting of $D$-level systems, the complementary reduced states of any bipartition share the same spectrum. This follows from its Schmidt decomposition. Hence, if a $(n-k)$-body reduction $\varrho_{B}$ is maximally mixed, then its complementary reduced state $\varrho_{A}$ of size $k \geq\lfloor n / 2\rfloor$ has all $D^{(n-k)}$ nonzero eigenvalues equal to $\lambda=D^{-(n-k)}$. Thus, the reduced state is proportional to a projector,

$$
\varrho_{A}^{2}=D^{-(n-k)} \varrho_{A}
$$

This projector property alone is already enough to derive the following bounds on the existence of AME states:

$$
n \leq \begin{cases}2\left(D^{2}-1\right) & n \text { even }, \\ 2 D(D+1)-1 & n \text { odd }\end{cases}
$$

These bounds originate in work by Rains and were applied to AME states by Scott $[3,20]$. A proof using the projector property can be found in Appendix A in the Supplemental Material [29].

Further, by Schmidt decomposition, one sees that the full state $|\psi\rangle_{A B}$ is an eigenvector of the reduced state $\varrho_{A}$

$$
\varrho_{A} \otimes \mathbb{1}^{\otimes(n-k)}|\psi\rangle_{A B}=D^{-(n-k)}|\psi\rangle_{A B} .
$$

Accordingly, for an AME state having all $\lfloor n / 2\rfloor$-body reduced states maximally mixed, any $k$-body reduced state $Q_{(k)}$ with $\lfloor n / 2\rfloor \leq k \leq n$ fulfills relations (7) and (9).

Now, let us consider AME states of $n$ qubits. We decompose Eq. (9) in the Bloch representation using the reduced state $Q_{(k)}$ on the first $k=\lfloor n / 2\rfloor+1$ parties of a qubit AME state

$$
\frac{1}{2^{k}}\left(\mathbb{1}^{\otimes k}+P_{k}^{(1 \cdots k)}\right) \otimes \mathbb{1}^{(n-k)}|\psi\rangle=2^{-(n-k)}|\psi\rangle .
$$

Because all $\lfloor n / 2\rfloor$-body marginals are maximally mixed, $P_{j \leq\lfloor n / 2\rfloor}=0$. We obtain the eigenvector relations

$$
P_{k}^{(1 \cdots k)} \otimes \mathbb{1}^{\otimes(n-k)}|\psi\rangle= \begin{cases}3|\psi\rangle & n \text { even } \\ 1|\psi\rangle & n \text { odd }\end{cases}
$$


By accounting for combinatorial factors, similar relations can be obtained in an iterative way for all $P_{j \geq\lfloor n / 2\rfloor+1}^{(1 \cdots j)}$.

With these building blocks in place, we are in the position to solve the last open qubit case-the existence of a seven qubit AME state. In the following, we will combine the projector property of a five qubit reduced state $Q_{(5)}$ with the eigenvector relations for $P_{4}^{(1 \cdots 5)}$ and $P_{5}^{(1 \cdots 5)}$ to obtain a contradiction from the parity rule stated in Lemma 1.

Observation 2.-Consider a pure state of seven qubits. Then, not all of its three-body reduced density matrices can be maximally mixed.

Proof-_Assume we have a pure seven-qubit state $\varrho=|\phi\rangle\langle\phi|$, whose three-body marginals are all maximally mixed. Then, its five-party reduced density matrix on systems $\{1, \ldots, 5\}$ is proportional to a projector

$$
\varrho_{(5)}^{2}=\frac{1}{4} \varrho_{(5)} \text {. }
$$

Note that, while the proof requires the projector property only to hold on the first five qubits, Eq. (12) actually holds for all possible five-qubit reductions.

Regarding the eigenvector relations, a Schmidt decomposition of the pure state $|\phi\rangle$ across the bipartitions $\{1,2,3,4 \mid 5,6,7\}$ and $\{1,2,3,4,5 \mid 6,7\}$ yields

$$
\begin{aligned}
& \varrho_{(4)} \otimes \mathbb{1}^{\otimes 3}|\phi\rangle=\frac{1}{8}|\phi\rangle, \\
& \varrho_{(5)} \otimes \mathbb{1}^{\otimes 2}|\phi\rangle=\frac{1}{4}|\phi\rangle .
\end{aligned}
$$

Again, analogous equations hold for any possible four- or five-qubit reductions, including the five different four-party reduced states in $\{1,2,3,4,5\}$.

We will use these three equations to obtain a contradiction: Let us expand $\varrho_{(4)}$ and $\varrho_{(5)}$ in the Bloch basis

$$
\begin{gathered}
\varrho_{(4)}=\frac{1}{2^{4}}\left(\mathbb{1}+P_{4}\right), \\
\varrho_{(5)}=\frac{1}{2^{5}}\left(\mathbb{1}+\sum_{j=1}^{5} P_{4}^{[j]} \otimes \mathbb{1}^{(j)}+P_{5}\right) .
\end{gathered}
$$

There are five different terms $P_{4}^{[j]} \otimes \mathbb{1}^{(j)}$, with $[j]$ indexing the five different supports of weight four terms within a five body reduced state, each having an identity on different positions.

Inserting Eqs. (15), (16) into Eqs. (13), (14) results in the eigenvector relations

$$
\begin{gathered}
P_{4}^{[j]} \otimes \mathbb{1}^{\otimes 3}|\phi\rangle=1|\phi\rangle, \\
P_{5} \otimes \mathbb{1}^{\otimes 2}|\phi\rangle=2|\phi\rangle .
\end{gathered}
$$

Similarly, we insert Eq. (16) in Eq. (12) to obtain

$$
\begin{aligned}
(\mathbb{1} & \left.+\sum_{j=1}^{5} P_{4}^{[j]} \otimes \mathbb{1}^{(j)}+P_{5}\right)\left(\mathbb{1}+\sum_{j=1}^{5} P_{4}^{[j]} \otimes \mathbb{1}^{(j)}+P_{5}\right) \\
& =8\left(\mathbb{1}+\sum_{j=1}^{5} P_{4}^{[j]} \otimes \mathbb{1}^{(j)}+P_{5}\right) .
\end{aligned}
$$

Now, the key observation is the parity rule stated in Lemma 1: Only certain products occurring on the left-hand side of Eq. (18) can contribute to $P_{5}$ on the right-hand side. Indeed, $P_{5}^{2}$ on the left-hand side cannot contribute to $P_{5}$ on the right-hand side. Similarly, $\left(\sum_{j=1}^{5} P_{4}^{[j]} \otimes \mathbb{1}^{(j)}\right)^{2}$ on the lefthand side cannot contribute to $P_{5}$ on the right-hand side.

Thus, we can collect terms of weight five on both sides of the equation,

$$
\left\{P_{5}, \sum_{j=1}^{5} P_{4}^{[j]} \otimes \mathbb{1}^{(j)}\right\}=6 P_{5} .
$$

Tensoring with the identity and multiplying by $|\phi\rangle$ from the right leads to

$$
\left\{P_{5}, \sum_{j=1}^{5} P_{4}^{[j]} \otimes \mathbb{1}^{(j)}\right\} \otimes \mathbb{1}^{\otimes 2}|\phi\rangle=6\left(P_{5} \otimes \mathbb{1}^{\otimes 2}\right)|\phi\rangle .
$$

However, using the eigenvector relations Eqs. (13), (14), one arrives at a contradiction

$$
(2 \times 5 \times 1+5 \times 1 \times 2)|\phi\rangle=6 \times 2|\phi\rangle .
$$

This ends the proof.

Upper bound for the number of maximally mixed reductions. - Note that, in the derivation above, not all constraints imposed by the reduced states have been taken into account. In fact, we only needed a single five-qubit reduced state (say, for definiteness, on the qubits $\{1,2,3,4,5\}$ ) fulfilling Eqs. (12), (14), whose three-body reduced density matrices are all maximally mixed [this was needed for Eq. (16)]. In addition, the five four-qubit reduced density matrices corresponding to the possible subsets of $\{1,2,3,4,5\}$ have to obey Eq. (13).

Thus, one can try to answer a relaxation of the original question: Given a seven-qubit state, whose two-party reduced states are all maximally mixed, how many of its three-party reduced states can then be maximally mixed? Consider a pure seven-qubit state where all two-body marginals are maximally mixed. This implies that any of the $\left(\begin{array}{l}7 \\ 5\end{array}\right)=21$ possible $Q_{(5)}$ obeys Eqs. (12), (14). There are $\left(\begin{array}{l}7 \\ 3\end{array}\right)=35$ possible $\varrho_{(3)}$ and corresponding $\varrho_{(4)}$. If a single three-qubit reduced state $\varrho_{(3)}$ (say, $\{1,2,3\}$ for definiteness) 


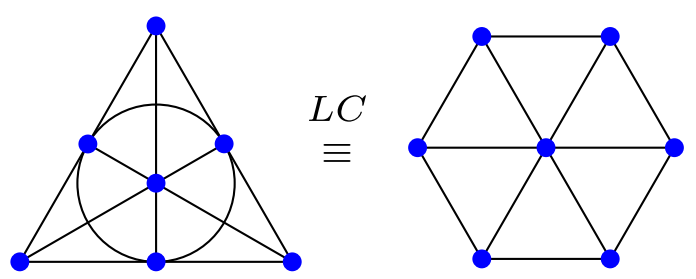

FIG. 1. The graph of the Fano (or seven-point) plane on the left, which can be transformed by local complementation (corresponding to local Clifford gates) to the wheel graph displayed on the right. The Fano plane plays a role in classical error correction, describing both a balanced block design as well as an error correcting code [31]. The corresponding graph state saturates the bound of Observation 3 . The states are locally equivalent to the graph state depicted in Figs. 1 in [30], to No. 44 in Table V from Ref. [19], and to the states of Eq. (11) in Ref. [9] and of Eq. (26) in Ref. [23].

is not maximally mixed, then nine of the $Q_{(5)}$ cannot be used for the proof anymore: First, for six five-qubit subsets (namely, $\{1,2,3,4,5\}, \ldots,\{1,2,3,6,7\}$ ) not all three-qubit density matrices are maximally mixed, implying that Eq. (16) is not valid. Furthermore, for three five-qubit subsets (namely, $\{1,4,5,6,7\},\{2,4,5,6,7\}$, and $\{3,4,5,6,7\}$ ) not all reduced four-qubit subsets obey Eq. (13). It follows that if two three-qubit reduced states are not maximally mixed, then at least $21-2 \times 9=3$ five-qubit sets still obey the conditions required for the proof. So we can summarize:

Observation 3.-Let $|\phi\rangle$ be a pure state of seven qubits, where all two-body reduced density matrices are maximally mixed. Then, maximally 32 of the 35 three-body density matrices can be maximally mixed. There exist seven-qubit states for which this bound is reached.

We note that the existence of states where 32 of the threebody density matrices are maximally mixed was shown before: References $[9,23]$ presented such states, which are, up to local unitary transformation, a graph state occurring in Refs. [19,30]. As a graph state, the state can be described by the graphs in Fig. 1. It can be constructed from the graph as follows: Each vertex in a graph corresponds to a qubit. One prepares all the qubits in the state $|+\rangle=(|0\rangle+|1\rangle) / \sqrt{2}$. Then, for any edge connecting the qubits $j$ and $k$, one applies a two-qubit phase gate

$$
C_{j k}=\left(\begin{array}{cccc}
1 & 0 & 0 & 0 \\
0 & 1 & 0 & 0 \\
0 & 0 & 1 & 0 \\
0 & 0 & 0 & -1
\end{array}\right)
$$

to the initial state. The fact that the marginals of this state have the right properties can also directly be checked in the stabilizer formalism, as explained in Ref. [19]. Finally, we add that there exists an AME state for seven threedimensional systems, which is also a graph state [13].
AME states of $n$ qubits.-The method presented for seven qubits can also be applied to the general $n$-qubit case. There, it can exclude that an AME state for a given number of qubits exists. It turns out that the qubit numbers $n$ for which no contradiction is found $(n=2,3,5,6)$ are exactly the ones for which AME states are known [15]. The proof is presented in Appendix B in the Supplemental Material [29].

Conclusion.-In summary, we have developed a method based on the Bloch representation for characterizing AME states. This allowed us to rederive most of the known results for qubits in a very simple manner, but more importantly, it solved the long-standing question regarding whether AME states of seven qubits exist or not. Also, the best approximation to such a state could be determined. For future work, it is very interesting to apply our methods to the question about whether $n$-qubit states exist where all $k$-body reduced density are maximally mixed for $k<\lfloor n / 2\rfloor$. These are not AME states, but they are central for quantum error correction and many efforts have been devoted to finding them in recent years $[32,33]$. We hope that our method can also contribute to solving this problem.

We thank Sara Di Martino, Jens Eisert, Christopher Eltschka, Mariami Gachechiladze, Dardo Goyeneche, Markus Grassl, Marcus Huber, Christian Majenz, and Karol Życzkowski for fruitful discussions. This work was supported by the Swiss National Science Foundation (Doc.Mobility Grant No. 165024), the COST Action MP1209, the FQXi Fund (Silicon Valley Community Foundation), the DFG, the ERC (Consolidator Grant No. 683107/TempoQ), the Basque Government Grant No. IT-472-10, Spanish Ministry of Economy, Industry and Competitiveness Grants No. FIS2012-36673-C03-01 and No. FIS2015-67161-P (MINECO/FEDER/UE), and the UPV/EHU Program No. UFI 11/55. Finally, we thank the Centro de Ciencias de Benasque Pedro Pascual and the Cafe Central in Innsbruck for hospitality.

[1] N. Gisin and H. Bechmann-Pasquinucci, Phys. Lett. A 246, 1 (1998).

[2] A. Higuchi and A. Sudbery, Phys. Lett. A 273, 213 (2000).

[3] A. J. Scott, Phys. Rev. A 69, 052330 (2004).

[4] I. D. K. Brown, S. Stepney, A. Sudbery, and S. L. Braunstein, J. Phys. A 38, 1119 (2005).

[5] A. Borras, A. R. Plastino, J. Batle, C. Zander, M. Casas, and A. Plastino, J. Phys. A 40, 13407 (2007).

[6] P. Facchi, G. Florio, G. Parisi, and S. Pascazio, Phys. Rev. A 77, 060304 (2008).

[7] P. Facchi, G. Florio, U. Marzolino, S. Pascazio, and G. Parisi, J. Phys. A 43, 225303 (2010).

[8] G. Gour and N. R. Wallach, J. Math. Phys. (N.Y.) 51, 112201 (2010).

[9] X.-W. Zha, H.-Y. Song, J.-X. Qi, D. Wang, and Q. Lan, J. Phys. A 45, 255302 (2012).

[10] W. Helwig, W. Cui, J. I. Latorre, A. Riera, and H.-K. Lo, Phys. Rev. A 86, 052335 (2012). 
[11] L. Arnaud and N. J. Cerf, Phys. Rev. A 87, 012319 (2013).

[12] W. Helwig and W. Cui, arXiv:1306.2536.

[13] W. Helwig, arXiv:1306.2879.

[14] C. Klöckl and M. Huber, Phys. Rev. A 91, 042339 (2015).

[15] D. Goyeneche, D. Alsina, J. I. Latorre, A. Riera, and K. Życzkowski, Phys. Rev. A 92, 032316 (2015).

[16] M Enríquez, I. Wintrowicz, and K. Życzkowski, J. Phys. Conf. Ser. 698, 012003 (2016).

[17] L. Chen and D. L. Zhou, Sci. Rep. 6, 27135 (2016).

[18] A. Bernal, Quant. Phys. Lett. 6, 1 (2017).

[19] M. Hein, J. Eisert, and H. J. Briegel, Phys. Rev. A 69, 062311 (2004).

[20] E. M. Rains, IEEE Trans. Inf. Theory 44, 1388 (1998).

[21] E. M. Rains, IEEE Trans. Inf. Theory 45, 2361 (1999).

[22] G. Nebe, E. M. Rains, and N. J. A. Sloane, Self-Dual Codes and Invariant Theory (Springer, Berlin, 2006).

[23] D. Goyeneche and K. Życzkowski, Phys. Rev. A 90, 022316 (2014).

[24] M. Grassl and M. Rötteler, in IEEE International Symposium on Information Theory (ISIT) (IEEE, Hong Kong, 2015), pp. 1104-1108.
[25] A. R. Calderbank, E. M. Rains, P. W. Shor, and N. J. A. Sloane, IEEE Trans. Inf. Theory 44, 1369 (1998).

[26] Up to $n=30$, the existence of one-dimensional nonadditive codes with parameters $((13,1,6))_{2},((19,1,8))_{2}$, and $((25,1,10))_{2}$ is still unresolved. See Table 13.3 in [22].

[27] U. Fano, Rev. Mod. Phys. 29, 74 (1957).

[28] C. Eltschka and J. Siewert, Phys. Rev. Lett. 114, 140402 (2015).

[29] See Supplemental Material at http://link.aps.org/ supplemental/10.1103/PhysRevLett.118.200502 for the Appendices.

[30] M. Grassl, A. Klappenecker, and M. Rötteler, in Proceedings IEEE International Symposium on Information Theory (IEEE, Lausanne, 2002), p. 45.

[31] R. Hill, A First Course in Coding Theory (Oxford University Press, Oxford, 1983).

[32] For a current overview on this problem see M. Grassl, Bounds on the minimum distance of linear codes and quantum codes, available at www.codetables.de.

[33] K. Feng, L. Jin, C. Xing, and C. Yuan, arXiv:1511 .07992 . 